\title{
Estrategias de Autocuidado en Equipos Profesionales que Trabajan en Maltrato Infantil
}

\section{Selfcare Strategies in Professional Teams That Work in Child Maltreatment}

\author{
Alejandra Isabel Santana \\ Universidad Santo Tomás
}

\author{
Chamarrita Farkas \\ Pontificia Universidad Católica de Chile
}

\begin{abstract}
Trabajar profesionalmente en maltrato infantil, implica asumir múltiples impactos, asociados a la temática y a la organización de la tarea. Esto ha llevado a crear Estrategias de Autocuidado para enfrentar esta situación. El objeto del estudio fue identificar las estrategias de los profesionales, a nivel individual y de equipo, y conocer la percepción de efectividad de éstas. Se utilizó una metodología cualitativa. La muestra de este estudio estuvo conformada por equipos interdisciplinarios y profesionales. Se utilizaron entrevistas y el análisis de los datos se realizó según la Grounded Theory. Los participantes han desarrollado las siguientes estrategias: individuales extra-laborales y laborales; de equipo, recreativas y laborales. En la percepción de efectividad, éstas se reconocen efectivas en su mayoría. Aún cuando la percepción de ineficacia se sustenta en la autocrítica de éstas.
\end{abstract}

Palabras Clave: autocuidado, maltrato infantil, equipos profesionales.

\begin{abstract}
Working professionally in child maltreatment, implies to assume multiple impacts, associate to the thematic and the organization of the task. This implies the creation of Self-care Strategies to face this situation. The aim of this study was to identify the strategies of professionals of this area, at individual and team level, and to investigate the perception of effectiveness of these. A qualitative methodology was used. The participants were professional teams. Interviews were used and the analysis of the data was carried out according to the Grounded Theory. They have developed the following strategies: individual extra-labor and labor strategies; team, recreational and labor strategies. Most of the strategies used are perceived as effective. However, the perception of inefficiency of these strategies is supported in the self-criticism of those.
\end{abstract}

Keywords: selfcare, child maltreatment, professional teams.

\section{Antecedentes}

\section{Equipos de Trabajo Interdisciplinarios en Maltrato Infantil}

En Chile (Sename, 2002), al igual que otros países del mundo (Australia, Bélgica, España), la intervención en maltrato Infantil ha sido organizada a través de equipos de trabajo interdisciplinarios ${ }^{1}$, cuyo abordaje generalmente, responde a intervenciones ecosistémicas de carácter psicosocial (Barudy, 1999;

Alejandra Santana López, Escuela de Trabajo Social, Universidad Santo Tomás, Chile.

Chamarrita Farkas, Escuela de Psicología, Pontificia Universidad Católica de Chile.

La correspondencia relativa a este artículo debe ser dirigida a Alejandra Santana, Gaspar de Orense 465, Quinta Normal, Santiago, Chile. E-mail: asantana@uct.cl

Disponible en Servicio Nacional de Menores, 1 de Junio 2002. Maltrato infantil (En línea). En http: //www.sename. $\mathrm{cl} / \mathrm{maltratol} /$
Morales \& Lira, 2000). Estos equipos, se constituyen como tales en cuanto responden a un trabajo interdependiente, actuando como "equipo de tareas" buscando desarrollar un estado de cooperación denominado "trabajo en equipo". Es decir, un grupo cooperativo en contacto regular que realiza una acción coordinada (Hidalgo \& Aylwin, 1992).

Por su parte, el adjetivo "interdisciplinario" hace referencia a la especialización de algunas disciplinas y el aumento de la complejidad de los fenómenos sociales, lo que ha llevado a la necesidad de abordar ciertas problemáticas, desde una mirada holística que vea más allá de las parcialidades, es decir, interdisciplinariamente.

Barudy $(1998,1999)$ hace referencia a los distintos niveles de especialización de estos equipos, planteando la organización en red de los sistemas profesionales, en lo concerniente a prevención y tratamiento del maltrato. A partir de esto, "propone un modelo piramidal de organización de una comunidad, donde el primer nivel corresponde a la mayor 
especialización y el quinto, al menos especializado en la gestión de casos de maltrato ${ }^{2}$ " (Barudy, 1999, p. 182).

Para efectos de este estudio, resulta interesante especificar la organización del primer nivel. Este, corresponde al Equipo Especializado:

encargado de la formación y coordinación de los niveles dos y tres, con el fin de movilizar los recursos profesionales de esos niveles para la gestión de situaciones de maltrato y acciones preventivas. El equipo especializado tiene como misión específica la validación y el tratamiento de las consecuencias del maltrato en sus diferentes formas, ya sea en sus aspectos médicos, psicológicos, relacionales y sociales que, por su complejidad y gravedad, no puedan ser tratados en otros niveles. Por su grado de especialización, este nivel tiene además la responsabilidad de desarrollar investigaciones sobre las causas y consecuencias de los diferentes tipos de maltrato, así como sobre la eficacia de los modelos de tratamiento y prevención. (Barudy, 1999, p.182)

Este modelo piramidal se complementa con la organización de atenciones en maltrato infantil, a través de circuitos de atención. Distinguiendo, circuitos de atención directa y circuitos de atención indirecta o asociada.

El circuito de atención directa se define por las funciones públicas asociadas, legal y directamente, a uno o más aspectos de la atención al maltrato infantil. Por su parte, el circuito de atención indirecta dice relación con las instituciones públicas que, por sus funciones con niños, tienen un rol importante ya sea en la prevención del maltrato infantil o en su detección y rehabilitación (Larraín, Vega \& Delgado, 1997).

A través de acción sincronizada de los circuitos, la política de infancia en el área de maltrato infantil se propone llevar a cabo un conjunto de acciones orientadas a restituir los derechos que han sido vulnerados a niños, niñas y adolescentes y contribuir al proceso reparatorio de una grave vulneración de derechos de la niñez. Dicho accionar, además de la intervención directa con los niños y niñas afectados por la vulneración de derechos, considera el

2 Niveles de la organización: nivel 1, equipo especializado facilitador y coordinador del proceso; nivel 2, profesionales de equipos pediátricos, salud mental y psicología escolar; nivel 3, profesionales de la salud atención primaria; nivel 4 , recursos de la comunidad; y nivel 5 , la comunidad. fortalecimiento de las capacidades y competencias parentales, los recursos familiares, sociales y comunitarios, con el objetivo de lograr su bienestar psicosocial ${ }^{3}$.

\section{Impacto del Trabajo con Casos de Maltrato Infantil en los Equipos de Trabajo Interdisciplinarios}

Asumiendo que el maltrato infantil es una de las expresiones de la violencia, tanto intra-familiar como de otros ámbitos sociales, es posible señalar que los equipos interdisciplinarios que intervienen con casos de maltrato infantil, al igual que en otras intervenciones con distintas expresiones de violencia, que implican alta carga emocional, viven desde sus procesos grupales ansiedades características de la intervención en violencia.

Pues, trabajar con las distintas manifestaciones de la violencia implica, en quienes interactúan directamente con los implicados -sean estos víctimas o victimarios- desgaste profesional, aún cuando el abordaje de la problemática sea como equipo y éste constituya un soporte para los profesionales en términos individuales; puede llegar a desgastarse también, dadas las temáticas abordadas y las dinámicas que se generan al interior de éstos (Morales \& Lira, 1996).

De hecho, "quienes trabajan con víctimas de cualquier tipo de violencia están expuestos a un nivel de desgaste profesional, que puede llegar al agotamiento profesional o burnout ${ }^{4}$ y ser la causa de trastornos psicológicos graves, del abandono de la profesión o del abandono del campo de trabajo (...) estas tendencias impactan a los grupos de trabajo, erosionándolos con graves consecuencias para su supervivencia como equipo, para sus integrantes y para quienes los consultan" (Arón, 2001, p. 67).

Los efectos producidos en el profesional que interviene en violencia, incluyen aspectos dados por la relación misma de persona atendida y profesional, pues las personas que han experimentado vivencias traumáticas provocan -en el caso particular de los terapeutas- un fuerte impacto subjetivocontratransferencial. En relación a éste, cabe seña-

\footnotetext{
3 Disponible en Servicio Nacional de Menores, 15 de Julio de 2004. Maltrato infantil (En línea). En http: // www.sename.cl/maltratol/

4 Término que nace desde el vocablo popular, haciendo metáfora al "fundimiento o queme profesional", la persona llega a un punto culminante de agotamiento, sin poder volver atrás (Maslach, 1982).
} 
lar que es un aspecto poco estudiado y escasamente abordado, "como si el hecho de trabajar con los efectos de experiencias de agresión, violencia y amenaza no dejara huellas" (Kovalskys \& Gómez, 2000, p. 317).

Este impacto en los profesionales ha ido recibiendo diversas denominaciones, así algunos autores hablan de: estrés traumático secundario, burnout profesional o riesgos de equipo.

El estrés traumático secundario, es un fenómeno de aprendizaje de otros traumas (...) el natural comportamiento y emociones generado por conocer eventos traumáticos experimentados por otros significativos (...) estrés resultante de ayudar o querer ayudar a personas traumatizadas o sufrientes (Figley, 1995 citado en Cornille \& Meyers, 1999). Es reconocido como un proceso a través del cual experiencias internas de los terapeutas son transformadas negativamente a través de un compromiso empático con el material de trauma de los clientes (Cornille \& Meyers, 1999).

Este concepto permite nombrar el estrés posttraumático ${ }^{5}$ sufrido por terapeutas o cualquier otro profesional o trabajador involucrado en el manejo de situaciones altamente estresantes y/o traumatizantes, "englobando todas las manifestaciones que pueden sufrir las personas relacionadas directa o indirectamente con sujetos víctimas primarias de trauma y pueden contener en su proceso de desarrollo algunas formas de contratransferencia ${ }^{6}$, trastornos de estrés post-traumático, burnout y victimización vicaria ${ }^{7}$ "(Paggi \& Gens, 2003, p.209).

Otra conceptualización que aborda los riesgos laborales asociados al trabajo en situaciones de ayuda, es la de Burnout. Éste es entendido como un cansancio físico y emocional, que involucra el desarrollo de una desvalorización del autoconcepto y el surgimiento de actitudes negativas hacia el trabajo, así como también pérdida de preocupación o emo-

\footnotetext{
5 Entendiendo por Estrés Post-traumático “el desarrollo de síntomas característicos que siguen a un acontecimiento psicológicamente traumático que por lo general se encuentra fuera del marco normal de la experiencia humana" (Paggi \& Gens, 2003, p. 202).

6 Entendiendo por Contratransferencia "el conjunto de reacciones inconscientes del analista frente a la persona del analizado y, especialmente frente a la transferencia de éste" (Paggi \& Gens, 2003, p. 206).

7 Entendiendo Traumatización vicaria como una "tensión permanente padecida por quienes están expuestos de manera crónica y aguda al trauma, a la congoja y a otras reacciones que son comunes en las víctimas" (Giberti, 2003, p. 232).
}

ción en el trato con las personas a quienes beneficia a través del trabajo. Esta condición se presenta, fundamentalmente en personas que trabajan en áreas de ayuda y que viven en contacto directo y continuo con personas necesitadas de apoyo (Maslach, 1982).

Farber (1991) plantea que un elemento esencial y común del concepto de burnout, es el hecho de aplicarse sólo a trabajadores de servicios de ayuda, a aquellos que se utilizan a sí mismos como herramienta en el trabajo con otros; un segundo elemento distintivo, es la "inconsecuencia" percibida por los profesionales cuando sus esfuerzos por ayudar a otros han sido ineficaces, dada la falta de retroalimentación por parte de los sistemas asistenciales. De hecho, Giberti (2003) identifica como primera zona de riesgo de burnout: "el ingreso en la actividad profesional incorporándose en diversas instituciones dedicadas a la atención, el cuidado y la defensa de los seres humanos, a partir de una ilusión redentora impulsada por la idealización de dicha práctica profesional" (Giberti, 2003, p. 230).

Una de las conceptualizaciones que dan cuenta de este impacto específicamente en los equipos, es la de Riesgos de Equipo, entendiéndolos como aquellos elementos de la dinámica grupal que ponen en riesgo al equipo en el desarrollo de la tarea constituyente, que se expresan en ansiedades que emergen en el vínculo con los grupos hacia los cuales se dirige la intervención (Morales \& Lira, 1996). Se asocian fundamentalmente a las constelaciones polares de sentimientos y a los mecanismos de defensa grupal.

\section{El Autocuidado de los Equipos Interdisciplinarios}

El autocuidado (en adelante AC) se entiende como "el conjunto de estrategias de afrontamiento que pueden implementar...(psicoterapeutas) ... para prevenir déficit y propiciar o fortalecer su bienestar integral (...) en las áreas: físicas, emocionales y psicoespirituales" (Murillo, 2001, p. 2).

Orem (1930 citada en Tapia \& Iturra, 1996), en el ámbito de la responsabilidad personal por la salud, sin distingo de profesión, centra el AC en la iniciativa propia de quienes lo requieren, entendiéndolo como "la práctica de actividades que los propios individuos, inician y desarrollan en su propio beneficio, en la mantención de su vida, salud y bienestar" (p. 6).

$\mathrm{El}$ AC tiene que ver con estrategias protectoras -sean éstas individuales, de equipo o institucio- 
nales-desplegadas por quienes trabajan directamente con víctimas de violencia; quienes ya han logrado reconocerse como profesionales y equipos en riesgo (Arón, 2001).

Barudy (1999) considera que la noción de AC posee dos niveles, el primero centra la responsabilidad en las instituciones, siendo éstas quienes primeramente protegen a sus recursos profesionales; el segundo nivel, se refiere a la capacidad de los profesionales de autocuidarse.

Cualquier programa que se declare adecuado en relación a la protección infantil debe tener en su interior un dispositivo para despertar, promover, mantener y proteger la implicación, ética y política de los profesionales. Esto, como el mejor antídoto "al síndrome del queme profesional" (...) todo programa o institución incapaz de proteger a sus profesionales, ejerce una doble violencia: a las personas de los profesionales $\mathrm{y}$, por ende, a los niños que protege. (Barudy, 1999, p.215)

\section{Modelos de Autocuidado}

Modelo de $A C$ desde la perspectiva del afrontamiento a través del apoyo social. Tonon (2003), desarrolla este modelo como una forma de afrontar el síndrome del burnout en profesionales dedicados a servicios y ayuda a otros a través del Apoyo Social en el trabajo. Entendiendo por Apoyo Social "todo proceso de transición interpersonal proveniente de los recursos sociales naturales o redes sociales de pertenencia, que potencian y favorecen el bienestar de los agentes implicados" (Martínez, García \& Maya, 2000 citado en Tonon, 2003). Es posible identificar tres tipos de apoyo: emocional, instrumental e informacional. El primero, se expresa en el sentimiento de ser amado, de poder contar con alguien, de poder ser escuchado. El segundo, consiste en la prestación de ayuda directa o de algún servicio; y finalmente, el tercero, consiste en brindar información, consejo y/o guía para la resolución de los problemas.

El apoyo social se materializa en el trabajo en las redes de apoyo social. Las redes sociales conforman el principal sistema de apoyo natural que tiene una persona.

El apoyo social en el trabajo aumenta la realización personal en el trabajo, disminuye el agotamiento emocional y mejora las actitudes y conductas negativas del sujeto hacia los demás (Gil Monte \& Peiró, 1997 citado en Tonon, 2003). Este apoyo puede provenir de diferentes fuentes: los compañeros de equipo, los grupos informales y hasta los jefes. En el contexto laboral es importante diferenciar la red de apoyo que conforman los otros miembros de la organización con quienes el profesional comparte el espacio de trabajo cotidiano, de los amigos con quienes pudiera contar.

Esta forma enuncia estrategias generales para abordar el problema del burnout, las cuales se sustentarían en la conceptualización de apoyo social y su operativización a través de las redes de apoyo social, concretamente laborales (Gil Monte \& Peiró, 1997 citado en Tonon, 2003):

- Revisar las modalidades de trabajo cotidiano.

- Rediseñar las tareas.

- Distanciamiento mental del trabajo fuera del horario laboral.

- Contar con momentos de descanso en el trabajo.

- Fijarse objetivos de trabajo reales y posibles de alcanzar.

- Aumentar la competencia profesional a partir del desarrollo de la capacitación.

Modelos de AC de Morales y Lira. En relación a los equipos psicosociales que abordan el maltrato, Morales y Lira (2000) han sistematizado una experiencia en Chile que intenta dar cuenta de una "receta de AC" formulada desde estos mismos equipos, aplicando un taller de trabajo a cuarenta personas representantes de diversas regiones del país. Cabe destacar que ya se había realizado esta misma experiencia en contextos institucionales y culturales muy distintos, tales como Perú (1997), Turquía (1996) y Angola (1998).

En base a la recopilación de Talleres de Trabajo en los distintos contextos señalados, se logran identificar cuatro Modelos de AC (Morales \& Lira, 2000):

a. Modelo de Reivindicación Institucional: este enfatiza en las condiciones institucionales en que se desempeña la labor del equipo. Dentro de éstas se encuentran: déficit de condiciones institucionales, escasa infraestructura física, inestabilidad laboral, bajos salarios, ausencia de previsión y salud para los trabajadores de los equipos.

b. Modelo de Identidad Social: éste se refiere a todos aquellos aspectos que hacen referencia al rol adjudicado y/o adjudicable, tanto a los programas que trabajan en situaciones de violencia como a las identidades gremiales y/o profesionales de sus componentes. Dentro de éstas se encuentran las necesidades de diferenciación y reconocimiento del valor de la labor realizada del programa frente a la institucionalidad, la diferen- 
ciación al interior de los equipos por profesión y experiencia.

c. Modelo de Impacto Emocional: se refiere a todos aquellos aspectos transferenciales y contratransferenciales del trabajo con personas afectadas por la violencia, que implica el manejo y cuidado respecto de las emociones de los terapeutas. Dentro de éstas se encuentra la necesidad de legitimar los sentimientos rabiosos hacia los pacientes, dar cuenta de las agresiones que los pacientes ejercen hacia los terapeutas y equipos, las dificultades de contención del sufrimiento y los límites de ésta, y todo aquello que algunos han llegado a denominar traumatización secundaria.

d. Modelo de Sociabilidad: este se refiere a los aspectos lúdicos, sociales y recreativos de los equipos. Dentro de estos se encuentran la necesidad de establecer espacios y ritos de esparcimiento y fundamentalmente de pertenencia grupal a la vida informal del equipo, como participación en los cumpleaños, apoyo en situaciones sociales o de salud de familiares del profesional, celebraciones, que a veces gatillan la disolución de límites o las autolimitaciones.

Modelo organizativo de programa de $A C$ de profesionales. En términos operativos, Jorge Barudy (1999) hace mención a este modelo, generado a partir de su participación en experiencias de equipos que han generado $\mathrm{EAC}^{8}$. Este tipo de Programa se basa en dos recursos fundamentales:

a. El altruismo social. La protección y el cuidado de los niños no es sólo el resultado de la competencia parental, sino el producto del esfuerzo de los padres, la familia y toda la sociedad.

b. La organización en redes. La eficacia y la competencia profesional depende de la capacidad de éstos de organizarse en redes.

El desarrollo de redes profesionales sanas debe ser considerado también como instrumento básico para evitar la fatiga profesional.

Las características de una red profesional que garantiza el AC de sus miembros son: coherencia interna, plasticidad estructural y capacidad de asociación.

\footnotetext{
8 En Programas como: Colectivo de Prevención del Sufrimiento Infantil (COPRES) en Bruselas; Servicio de la Infancia de la Diputación de Guipúzcoa, San Sebastián; Programa desarrollado por la Coordinación Social de Waremme, Bélgica; Programa del Departamento de Bienestar Social Foral de Alava, Vitoria; y Programa de la Asociación Vínculos, Andalucía.
}

- Coherencia interna: Mantención de su coherencia como organismo complejo, a pesar de las múltiples dificultades que la tarea le impone.

- La plasticidad estructural: La flexibilidad permite seguir desarrollando la tarea en forma coherente, a pesar de la complejidad y heterogeneidad de las situaciones de maltrato, así como la de las familias que lo producen.

- Capacidad de asociación: El ser humano es capaz de crear comportamientos asociativos, que organizados en forma ritualizada permiten mantener esta asociación en forma permanente y, al mismo tiempo, lograr producir palabras y discursos destinados a crear un sentido de pertenencia y cohesión, generando a sí un sentimiento de equipo y permitiendo mantener al grupo organizado a largo plazo alrededor de una tarea.

\section{Método}

\section{Participantes}

Los participantes de este estudio fueron profesionales y equipos pertenecientes a cuatro Centros de Atención Infanto Juvenil de la Corporación OPCIÓN ${ }^{9}$ ubicados en la Región Metropolitana, dos de ellos denominados Centro de Protección Infanto Juvenil (CEPIJ) y los otros dos, llamados Centro de Diagnóstico Ambulatorio (CODA).

En relación a los Centros, cabe destacar que:

- Los CEPIJs ${ }^{10}$ están conformados por equipos profesionales interdisciplinarios, los que trabajan en la reparación del daño psicosocial que afecta a niños y niñas víctimas de abuso sexual y situaciones de maltrato grave y leve de acuerdo a los criterios establecidos por el Ministerio de Justicia.

- Los CODAs ${ }^{11}$ están conformados por equipos profesionales interdisciplinarios, su labor se centra en la elaboración de diagnósticos e intervenciones psicosociales, si bien, abordan una amplia gama de problemáticas familiares vinculadas a la vulneración de los derechos de la infancia, se reconocen dentro de sus logros los avances en el abordaje terapéutico en maltrato infantil y abuso sexual en sectores de extrema pobreza.

En relación a los profesionales participantes en este estudio, éstos fueron mayoritariamente, asistentes sociales ( 8 a través de las entrevistas individuales y 13 en las entrevistas grupales de los equipos) y psicólogos ( 8 a través de las entrevistas individuales y 11 en las entrevistas grupales de los equipos, participando eventualmente 2 educadoras comunitarias (ambas en las entrevistas grupales).

9 OPCIÓN es una corporación privada sin fines de lucro que trabaja en el ámbito de los Derechos Humanos, diseñando, ejecutando y sistematizando programas de atención a la infancia adolescencia en las áreas de promoción y protección de derechos e infracción de la ley penal (http:// www.opcion.cl/presentacion1.html)

${ }^{10}$ OPCIÓN (2002).

11 OPCIÓN (2002). 
Respecto a los participantes se puede evaluar como una limitación del estudio que esta investigación se centró en cuatro centros pertenecientes a la Corporación OPCIÓN, entidad que posee un marco valórico explícito, en el cual se revelan los derechos de las personas y el respeto por los profesionales en su calidad de trabajadores, lo que podría aparecer como un facilitador a la hora de implementar y formalizar acciones de AC. Por lo tanto, no es posible generalizar que este tipo de desarrollo en $\mathrm{AC}$ se encuentre en otros equipos que aborden maltrato desde otros referentes valóricos.

\section{Materiales e Instrumentos}

Los instrumentos utilizados en este estudio fueron: Entrevista en profundidad individual y Entrevista en profundidad grupal.

La entrevista cualitativa en profundidad corresponde a "reiterados encuentros cara a cara entre el investigador y los informantes, encuentros dirigidos a la comprensión de las perspectivas que tienen los informantes respecto de sus vidas, experiencias o situaciones, tal como las expresan con sus propias palabras" (Taylor \& Bogdan, 1998, p. 101).

Las entrevistas individuales fueron dirigidas a los profesionales y estuvieron guiadas por las preguntas directrices del estudio.

Las entrevistas en profundidad de carácter grupal, corresponden a una variación de la entrevista en profundidad, y cuentan con la riqueza adicional de permitirle al investigador en un espacio y tiempo común, entrevistar a varios participantes, esto implica captar las dinámicas de las interacciones de ellos y sus posiciones respecto a los temas que en la sesión se traten.

Cabe destacar que las guías temáticas que condujeron ambas entrevistas fueron chequeadas a través de la realización de dos entrevistas (una individual y una grupal) de prueba a un equipo profesional que trabaja en una temática semejante. Permitiendo clarificar las preguntas y mejorar la organización del tiempo.

\section{Diseño y Procedimiento}

En el presente estudio se optó por un diseño descriptivo-analítico de carácter cualitativo, privilegiando una aproximación a las cualidades del objeto de estudio más que a sus cantidades, pues este enfoque "produce datos descriptivos: las propias palabras de las personas, habladas o escritas y la conducta observable" (Krause, 1996, p.2).

Específicamente la presente investigación corresponde a un Estudio exploratorio-descriptivo relacional, utilizando en su realización un Diseño emergente.

En relación a los procedimientos, se utilizó un muestreo intencionado a través de criterios de inclusión. Estos fueron: Centros de atención a la Infancia en Maltrato Infantil: leve, moderado, grave y/o abuso sexual; ubicados en la Región Metropolitana; que contaran con más de tres años de existencia; y que estuvieran dispuestos e interesados en participar de una estudio cuya temática fuera el AC. No obstante, se estableció preconcebidamente el número de Centros a abordar, bajo el criterio de viabilidad del estudio, al igual que el establecimiento un número mínimo de entrevistas individuales (4 por centro) y grupales (1 por centro).

Cabe destacar, que el tipo de muestreo se modificó respecto a cómo estaba contemplado inicialmente, constitu- yéndose en una limitación metodológica. En el diseño del estudio se había planteado un muestreo teórico, sin embargo, la dificultad para llevar a cabo en rigor este tipo de muestreo, llevó a cambiarlo por un muestreo intencionado a través de criterios de inclusión. Lo anterior por razones de viabilidad, limitaciones en los recursos temporales y humanos.

La forma en que se llevó a cabo la recolección de datos, contempló un primer momento en que la investigadora tuvo un acercamiento a los equipos profesionales interdisciplinarios, presentando el proyecto de investigación e invitándolos a participar.

Se tendió a realizar primeramente la entrevista grupal, con la finalidad de - a partir de sus contenidos- aportar temas más precisos a las entrevistas individuales que se realizarían posteriormente.

El análisis de datos se realizó por medio de un proceso inductivo que permitió construir categorías generales a partir de datos particulares. En este estudio, se realizó un análisis de significados y contenidos siguiendo los procedimientos de la Grounded Theory de Glasser y Strauss (1969, citado en Valles, 1999).

El método de análisis establece que se analiza palabra por palabra, frase por frase las entrevistas realizadas, siempre teniendo en cuenta la pregunta de investigación. El análisis de las entrevistas permitió elaborar categorías de diversos niveles de abstracción a partir de los datos, las que a medida que avanzó la investigación fueron observadas en relación a su nivel de generalidad para distintas entrevistas, también se consideró cómo dichas categorías se modifican conforme aparecían nuevos datos, etc. En la medida que el análisis de los datos se fue realizando y se fueron generando nuevas categorías y se detallaron relaciones entre éstas, 1legando a elaborar dos tipos de modelos de análisis: la codificación abierta y la codificación axial.

\section{Resultados}

\section{Análisis Cualitativo}

\section{¿Qué es el AC?}

"Es el espacio para estar atento de cuidar la propia integridad, lo que al individuo le está sucediendo con ese trabajo..." (VI, 2).*

Uno de los elementos que reportó mayor recurrencia, fue el responsabilizarse a sí mismo como principal agente de desarrollo del AC:

he sido responsable en el autocuidarme, fui capaz de poner límites... si no me habría quemado hace tiempo" (XIX, 26)...estar bien consigo mismo para poder estar bien con los demás, con el equipo y con las personas que uno atiende (XVII, 34).

El AC se vincula a la acción de cuidarse, protegerse y prevenir el daño ocasionado por el material

\footnotetext{
* Las siglas que acompañan las viñetas adjuntas a los discursos de los informantes corresponden a: el número romano identifica el número de Entrevista en que se extrajo la cita y el número arábigo señala el párrafo preciso de la cita al interior de esa entrevista.
} 
con el cual trabajan. En este caso, se asocia a la labor en maltrato infantil, pues se reconoce como un espacio laboral desde donde han surgido acciones específicas de AC, "el AC lo hemos desarrollado los equipos de maltrato para poder tener un espacio en donde podamos ver todos los casos que atendemos y que nos van afectando" (VIII, 2).

Esta protección - generada desde la experienciano sólo surge desde la motivación personal por cuidarse, sino que también está mediada por el interés de proteger el recurso que se posee para intervenir. Así, al indagar sobre qué los moviliza a preocuparse del $\mathrm{AC}$, es posible reconocer motivaciones personales a la base, asociadas a: querer realizar un trabajo de calidad y cuidarse a sí mismo en ese espacio.

Algunos profesionales ven el AC como proceso, donde surgen acciones que trascienden la responsabilidad personal, y lo vinculan a las interacciones dadas en los equipos y a las relaciones establecidas con la institución; así, se considera que "el AC no debería ser responsabilidad de una persona, debería ser de todos..." $(\mathrm{X}, 45)$.

Cabe destacar, que los profesionales otorgan al AC no sólo un valor "reparatorio" del daño al que se exponen en su trabajo, sino que también es validado como una instancia de prevención del queme profesional.

\section{Ámbitos de Acción Para el AC: Personal, Grupal e Institucional}

El ámbito de acción personal del $\mathrm{AC}$, es el más reconocido, lo que es coherente con la responsabilidad personal que se asignan en esta tarea, éste estaría mediado por las características personales del profesional, así como por su propia historia de vida, "darse cuenta en qué estás, porque uno está aquí, también porque elegí este tema y no otro, porque llevo tanto tiempo acá" (II, 8). Lo que sustenta este ámbito, estaría dado por una base valórica, donde cada cual asigna un valor distinto al trabajo, siendo para algunos sólo una dimensión de la vida y para otros, aspecto central de sus existencias. De esta dimensión dependerá gran parte de las Estrategias de $\mathrm{AC}$ (en adelante EAC) que los profesionales manifiestan para seguir desempeñándose en esta área laboral.

En el ámbito de acción grupal del AC se debe tener presente un antecedente imprescindible: la toma de conciencia de la posición de profesionales de alto riesgo, dada la exposición a una mayor vulnerabilidad, por condiciones propias de la tarea. Dentro de este ámbito, también se consideran las Relaciones Intraequipo, pues al asumir la tarea de intervenir en maltrato, ésta ya resulta violenta por su propia naturaleza, pero también les exige relacionarse de una manera no menos estresante, lo que muchas veces lleva a deteriorar las relaciones intraequipo; "implica tener una relación mejor con el resto del equipo, que aporte en todo sentido, que aporte el equilibrio..." (I, 2). Se destaca la voluntad e iniciativa de los equipos por trabajar el AC como tal.

El tercer ámbito de acción, lo institucional, se reconoce de suma importancia, pues - desde la perspectiva de los participantes- hasta ahora ha estado superficialmente implicado en el AC profesional, pese a ser considerada una dimensión clave, relevada a nivel de los discursos institu-cionales, traducida en intenciones de proveer de condiciones mínimas para desarrollar el AC en los equipos. "...hay una cierta idea de que es importante a nivel de la institución, sin embargo no es una política..." $(\mathrm{V}, 69)$, “... no hay plata destinada para esto" $(\mathrm{V}, 71)$.

\section{Estrategias de Autocuidado Según el Ámbito de Acción en que Surgen}

Para referirse a los tipos de EAC según ámbito de acción en que surgen, se abordaran dos grandes tipologías: aquellas de carácter personal y aquellas grupales.

Estrategias de AC personal. Las EAC de carácter personal se pueden subclasificar en Extralaborales (referidas a: Cuidado Personal, Actividades Sociales, Actitudes hacia la vida y/o trabajo, y Recreativas propiamente tal) y Laborales. En términos generales, se caracterizan por ser implementadas individualmente por el profesional y pudiendo o no desarrollarse en el espacio de trabajo.

Estrategias de AC personal extralaborales. Al interior de la Estrategias Personales Extralaborales, destacan aquellas referidas al cuidado personal, entre ellas: realizarse algún tipo de psicoterapia, referida a lo menos por la mitad de los participantes, “...lo pude superar, con mucho trabajo, psicoterapia, con tratamiento medicamentoso...eso me ayudó a preocuparme de mí..." (II, 34). Cabe destacar que el uso de la psicoterapia no sólo tiene una función "curativa", es decir, no sólo es buscada cuando el profesional se siente dañado sino que también se utiliza como proceso de ayuda permanente.

Otra estrategia, es el Darse Tiempo para sí Mismo(a), que está estrechamente relacionada con la señalada anteriormente, pero asume no sólo la dimensión psicológica del profesional sino que puede aborda otras esferas. 
En las Estrategias Personales Extralaborales referidas a Actividades Sociales, principalmente aquellas vinculadas a las redes sociales más próximas, destacan: el compartir en familia y la reactivación de redes sociales personales, donde los amigos asumen un rol central, asociándolo también a otros espacios recreativos, como salidas a comer y/o bailar. Estas instancias han sido un apoyo en lo referente a evitar contaminar esos espacios con temáticas laborales, “... ya no hablo de trabajo en otros espacios... vas a una reunión social y dices, por favor que no te pregunten por tu trabajo, a lo más dices, trabajo en un centro de orientación familiar y chao..." (XI, 12). Esta última estrategia está estrechamente ligada a actitudes hacia la vida y/o el trabajo, donde lo mayormente reportado y con mayor significación para los profesionales resultó ser el "evitar hablar de trabajo fuera de este ámbito".

Ahora bien, dentro de las Estrategias Recreativas propiamente tales, una de las más reconocidas es la práctica de algún deporte, “.... una práctica que he tenido ha sido la actividad física, siempre hago deporte, especialmente gimnasia, que en un período difícil me sirvió mucho (...) en cuanto a relajarme, a botar tensiones a través de la actividad física...” (I, 48). Este espacio, posee la riqueza de favorecer la desconexión de las preocupaciones propias del trabajo, descargar tensiones y reportar un bienestar físico asociado a su práctica.

Estrategias de AC personal laborales. Al interior de las Estrategias Personales de carácter Laboral, aquella que posee mayor mención, es el establecimiento de límites entre el trabajo y la vida personal, manifestado en "tomándome el trabajo como un trabajo no como un apostolado... uno está aquí para reparar y mientras yo pueda hacer este trabajo voy a estar bien..."(III, 22). A este respecto, el establecimiento de límites no se circunscribe a determinar qué es parte de la vida laboral y qué es parte de otras dimensiones, sino que también aborda la delimitación de funciones y tareas dentro de la institución. Es interesante recordar, que para muchos de los entrevistados esto sería una acción constituyente del concepto de AC.

Siguiendo con las Estrategias Personales Laborales, existen un conjunto de éstas que se involucran directamente con la tarea, teniendo como elemento común el usar la comunicación como sustento, dentro de ésta: el establecer temas de discusión, el recibir supervisión de los casos de los propios pares, tocar el tema del AC explícitamente, abordar diferencias en las relaciones del equipo, comunicarse permanentemente con la dupla, "... lo otro es conversar siempre con mi dupla después de las sesiones (...) no es un sistema, sino que en el fondo si yo la veo mal, le pregunto..." (VII, 28).

Otra estrategia de esta tipología, la constituyen el mejorar o hacer agradable el entorno físico en el cual se trabaja, “... me gusta preocuparme por las cosas del ambiente, concretamente que esté bonito, que sea agradable, que haya buenos materiales y hacer tareas físicas en las horas de trabajo, ya sea reparar juguetes o preparar materiales..." (IV, 32). En este sentido, según lo señalan los participantes, el hacer del ambiente de trabajo un espacio grato depende de los profesionales y es misión de ellos que esto ocurra, siendo una tarea cotidiana, que permite distraerse del trabajo en maltrato, y a la vez, da cuenta de cuánto se valora el lugar de trabajo, en tanto, agradable para sí y para los otros.

Una Estrategia Personal Laboral asociada a nutrirse en pro de mejorar las intervenciones es el recibir capacitación en el tema de maltrato infantil.

Resulta interesante señalar cómo el hecho de trabajar simultáneamente en otros lugares, con otras temáticas, lo que ha simple vista podría identificarse como una sobrecarga más, pasa a ser reconocida como una EAC.

Cabe destacar, que gran parte de los profesionales incluyó en sus EAC Personales Laborales, aquellas que son compartidas con el equipo. Las que serán abordadas a continuación.

\section{Estrategias de AC Grupal}

Otro tipo de EAC, son aquellas de carácter grupal, las cuales son llevadas a cabo por el equipo profesional, y pueden vincularse a la tarea directamente. Para los equipos participantes, las EAC grupales, poseen como características centrales: el no ser estructuradas, tendientes a la recreación, financiadas con recursos de los propios equipos y que intenten dar respuesta a necesidades sentidas por el equipo.

Estrategias de AC grupal recreativas. Dentro de las EAC grupal, sobresalen aquellas tendientes a la recreación en conjunto, para esto se destinan horas al mes, éstas pueden usarse en actividades netamente recreativas o al abordaje de temáticas asociadas a la tarea y al AC. Uno de los contenidos posibles de las horas de $\mathrm{AC}$, son aquellos de carácter lúdico, donde el juego es reconocido como una de las EAC fundamentales, “... trabajamos juntos, nos quedamos acá y a veces trabajamos ciertos temas, pero básicamente hacemos actividades de 
descompresión...” (XX, 10). También se identifica el compartir espacios recreativos. Éstos pueden o no situarse en el contexto laboral, pues incluirían la celebración de cumpleaños, el salir a comer o el salir fuera de la ciudad.

Otro espacio de recreación son los desayunos colectivos previos a la realización de la reunión técnica o contemplados dentro de la rutina semanal de trabajo, “... yo recuerdo que una de las primeras cosas que se instauró fue el desayuno colectivo que era previo a la reunión técnica, como un espacio de distensión, de compartir, de fraternizar...” (V, 54).

Estrategias de AC grupal asociadas a la tarea. Uno de los contenidos posibles a trabajar, dentro de las horas destinadas a $\mathrm{AC}$, son aquellos vinculados directamente con la tarea de intervenir en maltrato. En este ámbito, se identifica: la supervisión de los casos, el tratar temáticas referidas al AC-temas emergentes o vinculados a la descompresión- intentando guiarse por ciertos objetivos concretos. Una estrategia grupal, que tiene que ver con el espacio disponible para autocuidarse, lo constituyen las reuniones técnicas,

... también tenemos un supervisor (...) es un profesional que nos da su apoyo, es externo al proyecto, a la institución, que se financia con plata del proyecto y que viene dos veces al mes, participa en la reunión técnica y en algunas actividades de las que hacemos de AC, él además nos nutre desde su experticia y nos apoya...". (IX, 18)

Existen un grupo de EAC utilizadas por el equipo que se vinculan a la tarea pero no necesariamente se dan en las horas contempladas para AC, sino más bien, se despliegan en la medida que surge la necesidad de hacerlo, principalmente el descomprimirse con sus pares, ésta tiene un carácter eminentemente comunicacional y por lo tanto, relacional. Algunas expresiones de esto son: preocuparse por el otro si se ve mal, escuchar al otro, contenerse si es necesario, construir el espacio para desahogarse después de la sesión, socializar acerca del trabajo.

Al intentar indagar si los profesionales reconocen algunas EAC asociadas a algún riesgo específico, se evidenciaron, relaciones particulares, concluyendo básicamente en que no existe tal relación.

\section{¿Cuán eficaces son las EAC desde la perspectiva de los participantes?}

Un elemento crucial, a la hora de intentar evaluar -partir de los propios participantes- cuán eficaces son las EAC que se utilizan en la actualidad, son las percepciones de índole personal y de equipo respecto a las manifestaciones concretas del nivel de eficacia.

Percepción de eficacia de las EAC a nivel individual. En relación a las EAC Individuales, se reportaron expresiones de eficacia, siendo las principales manifestaciones de ella: el sentimiento de bienestar vivido...en términos de como me levanto en la mañana para venir al trabajo, mucho más animosa, no como una tortura..." (III, 35) “...me han funcionado (...) yo creo que en términos personales está bien, o sea, yo salgo de acá y estoy bien, me desconecto, y lo hago de verdad, no me engaño..." (XI, 24) ; la motivación por el trabajo, “... yo tengo una motivación permanente por trabajar con la gente, el trabajo en sesiones, en ir a terreno, en estar con la gente, su medio, en compartir más que intervenir, siento que estoy con ganas, no me siento derrotado, sin ganas de ir a trabajar... (II, 56) y el reconocer las estrategias como una ayuda.

Las estrategias individuales de carácter laboral, expresaron su eficacia a través de no seguir pensando en el trabajo fuera del horario de éste, “... cada vez más, se ha ido estructurando para darle efectivamente el rol de AC, que te proteja más. Yo no me quedo cargada con los casos, no me voy a mi casa pensando en ellos..." (XII, 12). Sin embargo, también se señala una manifestación de ineficacia expresada en el reconocimiento de la falta de desarrollo de este tipo de estrategias, "... se requieren procesos más metódicos, más orientados al AC propiamente tal, como equipo, como proyecto, $\mathrm{y}$ tomando en cuenta las temáticas porque concretamente yo soy parte de este equipo...” (I, 52), “... te dicen tienes medio día al mes de AC pero no te dicen nada más, o sea, qué hacer, qué herramientas o dinámicas, qué temas, no es una necesidad para ellos, no siento que haya disposición de ponerse frente al tema y otorgar personas que sepan del tema y que nos apoyen..." (XI, 24).

Percepción de eficacia de las EAC a nivel grupal. En las percepciones de eficacia señaladas por el equipo se reconocen variadas y recurrentes expresiones de eficacia, entre éstas: favorecimiento de la integración, "...es poco lo que podemos hacer por el tiempo y los recursos, pero yo creo que los carretes, las salidas, los desayunos y todo lo que hacemos permiten harta integración del grupo, harto conocerse en otras instancias..." (XVII, 28), mejora en las relaciones del equipo, especialmente a nivel comunicativo y fortalecimiento de elementos asociados directamente con la tarea, “... tenemos un 
buen nivel relacional, que podemos ver las señales en el otro de que están pasándole cosas...” (X, 8). En síntesis, se reconocen cambios positivos al interior de los equipos gracias al desarrollo de EAC.

Quienes señalan expresiones de ineficacias corresponden a relatos particulares, y por tanto, no recurrentes.

\section{Análisis Relacional}

\section{Relación AC y Estrategias de AC}

El AC como noción está elaborado por la totalidad de los profesionales que intervienen en maltrato infantil y se caracteriza por dar cuenta de una intencionalidad, de querer llegar a ese concepto construido por cada uno, con los matices que cada cual aporta; aún cuando la mayoría lo asocia a responsabilidad consigo mismo, con el establecimiento de límites, con la tarea de protegerse, visualizándolo como proceso y poseedor de dimensiones que lo constituyen, aparece más bien, como una aspiración.

Este concepto, no cobra vida hasta que es operacionalizado a través de acciones, pues el AC es por sobre todo acción y pro acción, es decir cuidarse de lo que daña en el trabajo y protegerse de aspectos vulnerables a los que es posible anticiparse. De este modo, el AC de despliega a través de estrategias, o sea, buscando distintos caminos que conduzcan al bienestar laboral.

No cabe duda, que todo los profesionales participantes, han desplegado EAC, aún cuando quizás -en momentos- no hayan tenido conciencia de lo que hacían, presentándose como acciones espontáneas que permitían afrontar la tarea y mantenerse en sus trabajos.

Para los participantes, el AC es un tema explícitamente abordado como equipo, por tanto, son capaces de identificar las estrategias particulares que han utilizado, y a la vez tienen la capacidad de analizarlas, emitir juicios de valor acerca de cómo se han conducido y proponer cambios que llevaría a una mejora.

Las EAC, al igual que el AC, tienen ámbitos de acción, personales y grupales, manifiestas en los espacios laborales y extralaborales, pero que apuntan a un mismo objetivo: estar bien íntegramente consigo mismo, con el entorno inmediato y realizar una labor de reparación en maltrato infantil sin el costo de autodestruirse en este intento.

El que sean identificadas las EAC, permite darse cuenta que los profesionales han estado trabajando este tema, probablemente en distintos niveles de desarrollo; algunos recién en la toma de conciencia de la situación de vulnerabilidad dada por la posición laboral y otros, pensando en programar acciones dentro de un plan formalizado de AC.

Lo anterior, permite que los profesionales sean capaces de aportar una mirada crítica al trabajo realizado en torno al AC, generando algunas propuestas de acciones. Si bien, éstas se pueden reconocer como demandas formuladas a una esfera que trasciende lo grupal, ya que la mayoría de las propuestas apuntan a destinar mayores recursos, contar con apoyo externo, implementar espacio de resguardo laboral para los profesionales en su condición de trabajadores, entre otras.

\section{Relación de Estrategias de AC y Percepción de Eficacia}

Para los participantes las acciones implementadas en pro del AC han resultado eficaces, lo que se ha reflejado en los indicadores que ellos mismo han identificado; en términos personales el sentimiento de bienestar, el seguir motivado y con ánimo para trabajar; y a nivel grupal, dando evidentes signos de fortalecimiento e integración del equipo, principalmente a través de la mejora en la comunicación.

Pese a esto, un número significativo señaló que éstas no son plenamente eficaces pues evalúan que aún queda camino por recorrer en lo que a desarrollo de $\mathrm{AC}$ se refiere, principalmente en la formalización y organización de las acciones tendientes a autocuidar a los profesionales, de tal modo que no sólo se centren en lo recreativo, sino que aborden de manera integral la persona del profesional.

Destaca que los principales indicadores de efectividad percibidos por los profesionales y equipos, los constituyan elementos intrínsecos a ellos mismos o que se vinculen a la forma y calidad de comunicación dada entre ellos, dejando fuera aquellos elementos propios de las condiciones de trabajo. Lo anterior es coherente con lo abordado en el apartado anterior, donde los profesionales y equipos no se vislumbran como protagonistas del cambio en los riesgos asociados a las condiciones laborales.

\section{Discusión}

Para los equipos participantes, el AC es un proceso tendiente al logro de bienestar físico y emocional, enfocado en la prevención del queme o en la reparación una vez que éste ya ha dado algunas 
señales; cuya responsabilidad recae directamente en la persona del profesional, aún cuando también poseen una cuota importante los equipos e instituciones donde se lleva cabo el trabajo con maltrato infantil. La referencia a la responsabilidad ya era enunciada por Barudy (1999), aún cuando éste sitúa la responsabilidad institucional en primer lugar, y a continuación la personal.

Las estrategias concretas de AC, desde lo personal, pueden clasificarse como extralaborales y laborales. Las primeras, se enfocan al cuidado personal del profesional, la activación de sus redes sociales más significativas - generalmente familia y amigos $-\mathrm{y}$ la realización de actividades recreativas propiamente tales. Las segundas, abordan principalmente la capacidad de establecer limites entre trabajo y vida personal, el dar y recibir supervisión por parte de los pares, favorecer una comunicación fluida con el resto del equipo, tender a hacer agradable el espacio físico de trabajo, recibir capacitación en el área, y dentro de lo posible, tener otro trabajo paralelo en algún área distinta a la de maltrato infantil.

Las EAC señaladas por los profesionales se presentan muy similares a las aportadas por Pearlman (1999) y sus estudios referentes a estrategias de enfrentamiento de profesionales que laboran en violencia doméstica y otras áreas de salud mental (Gamble, Pearlman, Lucca \& Allen, 1999; Follette, Polusny \& Milbeck, 1994; Schauben \& Frazier, 1999 citado en Pearlman, 1999), principalmente en las EAC extralaborales. Sin embargo, en estos estudios no se hace una valoración explícita por lo grupal como una EAC en sí misma, de contención, cooperación y comunicación permanente, al contrario de lo asumido por los equipos participantes de este estudio. De hecho, sólo en uno de ellos (Illiffe \& Steed, 2000) se hace mención al "apoyo de equipo" como una EAC, apuntando a evitar el aislamiento profesional.

Para los equipos participantes, las EAC desplegadas a nivel grupal pueden clasificarse en recreativas y asociadas a la tarea. Dentro de las estrategias recreativas destacó: compartir espacios recreativos, jugar, desarrollar un amplio sentido del humor y compartir desayunos colectivos.

Las estrategias grupales asociadas a la tarea, implican trabajar con horas destinadas al AC, las cuales pueden contener: supervisones de casos, abordaje explícito de algún contenido de AC propiamente tal, como por ejemplo, la descompresión.

En relación a las EAC grupal, destaca el hecho que éstas cobren relevancia en el contexto latinoamericano con mayor fuerza (Arón, 2001; Hidalgo \&
Moreira, 2000; Morales \& Lira, 2000) y que posean una crítica subyacente a las condiciones laborales macro en que se insertan los equipos. Situación, que no es mencionada en las EAC de los estudios norteamericanos y australianos referidos anteriormente. Si bien, los equipos de este estudio están abordando EAC particulares, recreativas y asociadas a la tarea cotidiana, sin embargo existe un malestar de fondo, latente, implícito, que podría mermar las iniciativas de AC desplegadas en la actualidad, con una suerte de "desesperanza", con el riesgo de quedarse a un nivel aparente y no de profunda responsabilidad por el cuidado de la integridad profesional.

Respecto a la eficacia de estas EAC, destaca que la metodología utilizada permitió acercarse a estas apreciaciones sin referentes preconcebidos de "eficacia" centrándose en lo que desde la percepción de cada cual, resultaba eficaz y en la manifestación de esto.

Tanto para los profesionales como para los equipos, las EAC utilizadas han resultado efectivas en relación a las necesidades que le dieron origen. De esta forma para los profesionales -desde lo personal- esta eficacia se manifiesta en: sentirse bien, motivados y animados para seguir trabajando, pese a esto, reconocen la necesidad de seguir trabajando y evolucionado estrategias de acuerdo a las particularidades de cada cual. Aquellas EAC personales extralaborales, resultaron totalmente efectivas, sin reportarse manifestaciones de "ineficacia"; por su parte, las EAC personales laborales, también fueron ampliamente eficaces, salvo quienes se centraron en la "ineficacia" como falta de desarrollo de este tipo de estrategia, es decir, más que encontrar estrategias "malas" se remiten a una "falta de desarrollo".

Como equipos reconocen la eficacia reflejada en la integración y fortalecimiento de elementos comunicacionales al interior del grupo; por su parte, la ineficacia es expresada como "falta" de herramientas, recursos y políticas de salud mental laboral, aun cuando se presentan en menor numero y recurrencia.

Resulta importante señalar, que la percepción evaluativa de los equipos también se reflejó al ubicar el desarrollo de EAC en distintos momentos del ciclo vital grupal, siendo capaces de discriminar estrategias inoperantes o que respondían a necesidades de contingencia en un tiempo pasado, y asumir nuevas estrategias, con otras características en un momento actual. Por ejemplo, estrategias tendientes a fortalecer el equipo en un momento de creación de éste o de renovación casi total, y estrategias que apunten a co-construir objetivos transversales de $\mathrm{AC}$ para el equipo, en un momento posterior. 
Cabe destacar, que si bien, es posible identificar eficaces EAC en equipos que trabajan en maltrato infantil, hay que ser cauteloso al creer que los equipos que trabajan en otras intervenciones psicosociales podrían asumirlas. Principalmente, porque las estrategias de equipo están estrechamente relacionadas a los procesos grupales, entendiendo al grupo como un suprasujeto de un sistema (Morales \& Lira, 1996), donde la voluntad, la dinámica interna, su estructura, su historia y sus crisis vitales, determinarán si se despliegan o no EAC y de qué características poseen éstas en caso de desplegarlas, sin olvidar que para estos equipos la tarea resultará el eje de su condición grupal (Pichón-Riviere, 1985 citado en Morales \& Lira, 1996). Por lo tanto, no se puede dar una "receta" de AC para todos los equipos que intervengan en maltrato, sino que se requerirá un primer momento de "diagnóstico de equipo", el cual debiera ser participativamente, de manera tal de sintonizar con sus prioridades y necesidades movilizadoras.

Al ubicar los equipos participantes y sus necesidades de AC insatisfechas, en los Modelos de AC señalados por Morales \& Lira (2000), se puede decir que éstos poseerían más satisfechos elementos de los Modelos de Impacto Emocional y Sociabilidad, en tanto, ámbitos que ya han sido abordados y trabajados con cierta frecuencia. No obstante, los Modelos insatisfechos o menos desarrollados serían, fundamentalmente el de Reivindicación Institucional e Identidad Social. El primero, expresado recurrentemente en la sobrecarga laboral, salarios no acordes a la tarea encomendada e inseguridad laboral. El segundo fue abordado, como una desventaja de trabajar en duplas psicosociales, temiendo perder la diferenciación entre profesionales. Además, las críticas y necesidades insatisfechas apuntan a condiciones laborales (modelo reivindicativo), las cuales son manifiestas con mayor potencia a través de los equipos en instancias grupales, lo cual también deja entre ver elementos identitarios (modelo de identidad social) como profesional del área psicosocial y como equipo interventor en maltrato infantil.

Ahora bien, desde lo operativo, Barudy (1999) ya proponía el trabajo en red como condición básica del AC de los profesionales. Sin embargo, de acuerdo a este estudio, el trabajo en red poseería vacíos que lo hacen en ocasiones inoperantes y por tanto, frustrante desde la perspectiva profesional. Dichos vacíos serían explicados, en parte, por las implicancias legales que poseen muchos casos de maltrato infantil, donde los tribunales como una red en sí misma funcionarían con criterios distintos a los de los profesionales interventores; a su vez, la red social de infancia no contaría con la suficiente promoción de sus instancias de protección para los niños, señalando algunos profesionales el desconocimiento de alternativas de derivación, a los escasos centros residenciales para niños en riesgo vital, con la idoneidad requerida, sin que sean nuevos espacios de maltrato y vulneración. De esta forma no se estaría contando con las características de la red que garanticen el AC de sus miembros, es decir, se contaría con un nivel deficiente de: coherencia interna, plasticidad estructural y capacidad de asociación.

De los Modelos de AC presentados, aquel que mejor refleja lo realizado en la práctica por los equipos interdisciplinarios, es el de Apoyo Social (Tonon, 2003). Fundamentalmente son emocionales e informacionales, aún cuando se demandaría por los participantes- un mayor apoyo experto y externo al centro. De acuerdo a este mismo Modelo, el equipo como grupo (Hidalgo \& Aylwin, 1992) constituye una red de apoyo. De esta forma, la apreciación de efectividad de las EAC grupales, sería coherente con lo planteado por Gil Monte \& Peirós (1997) reconociendo el apoyo social como fuente de realización personal, factor que permite disminuir el agotamiento emocional y mejora las actitudes hacia los demás.

Las experiencias que estos equipos poseen de $\mathrm{AC}$, dan pie a considerar que el trabajo sistemático de $\mathrm{AC}$ en los contextos laborales es viable, y que para esto requieren: la voluntad de los actores implicados y recursos para ello. Por su parte, el rol institucional debe orientarse a dar el espacio, y contar con la convicción de valorar este accionar como una inversión en valiosos equipos que se han consolidado a través de formación y de la experiencia cotidiana en maltrato. De esta forma, no sólo basta con dar "horas a AC" sino también es importante dar contenidos a ellas, lineamientos generales, pues ya se decía que cada equipo posee necesidades particulares; monitorear qué se está haciendo en $\mathrm{AC}$, con qué resultados, idear formas de difundir las experiencias y los aprendizajes generados a través de ellas, y por cierto evaluar la incidencia de un equipo "cuidado" en el desempeño de la tarea.

Ahora bien, otro elemento que permitiría y desarrollo de EAC pertinente y sostenido en el tiempo, es considerar las particularidades de cada equipo, fundamentalmente: la etapa del ciclo grupal en que 
se encuentran, las experiencias previas en maltrato y las necesidades sentidas en cuanto a AC. Para esto sería recomendable que el desarrollo de lineamiento de AC fuera desarrollado por los mismos equipos a través de una instancia participativa; lo que no significa que ellos mismos deban implementarlo, pues en ocasiones esta tarea se percibe como una carga más en el trabajo.

El presente estudio pone en evidencia la necesidad de los equipos por darle espacio a la salud mental laboral, y desplegar acciones concretas a favor del bienestar de la persona del profesional, resguardando su integridad en el desempeño de la labor.

También pretende motivar futuras investigaciones ligadas a la salud mental de profesionales que se desempeñan en el área psicosocial, dejando múltiples interrogantes. Ante todo abre la inquietud por implementar Programas de AC que recojan las necesidades básicas de los equipos y profesionales y que considere modificaciones al contexto de aparición y a las condiciones intervinientes generales.

\section{Referencias}

Arón, A. (2001). Violencia en la familia. Programa de intervención en red: La experiencia de San Bernardo. Santiago: Galdoc.

Barudy, J. (1998). El dolor invisible de la infancia. Buenos Aires: Paidós.

Barudy, J. (1999). Maltrato infantil. Ecología social: Prevención y reparación. Santiago: Galdoc.

Cornille, T. \& Meyers, T. (1999). Secondary traumatic stress among child protective service workers: Prevalence, severity and predictive. Journal of Traumatology. Recuperado el 15 de Abril de 2002, desde http://www.fsu.edu/ trauma/art2v5i1.htm

Davis, K. \& Newstrom, J. (2001). Comportamiento humano en el trabajo. México: Mac-Graw Hill.

Farber, B. (1991). Crisis in education: Stress and burnout in the American teacher. USA: Jossey-Bass Publishers.

Giberti, E. (2003). Atención a niños y niñas víctimas: El efecto burn-out en los profesionales. En S. Lamberti (Ed.), Riesgos del compromiso profesional. Buenos Aires: Universidad de Buenos Aires.

Hidalgo, C. \& Aylwin, N. (1992). Familia e interdisciplina. Documento de Trabajo 36. Programa de Postítulo de Estudios de la Familia. Santiago: Pontificia Universidad Católica de Chile \& Corporación de Promoción Universitaria.

Hidalgo, N. \& Moreira, E. (2000). Autocuidado, trastorno psicológico y rotación de equipos de salud mental. San- tiago: Departamento de Psicología, Universidad de Chile.

Iliffe, G. \& Steed, L. (2000). Exploring the counselor's experience of working with perpetrators and survivors of domestic violence. Journal of Interpersonal Violence, 15(4), 393-412.

Kovalskys, J. \& Gómez, E. (2000). Cultura de la violencia: $\mathrm{Su}$ impacto invisible en el paciente y en el vínculo terapéutico. En O. Vilchez (Ed.), Violencia en la cultura: Riesgos y estrategias de intervención. Santiago: Ediciones Sociedad Chilena de Psicología Clínica.

Krause, M. (1996). Apuntes primer curso de capacitación en técnicas cualitativas para equipos de alcohol y drogas del Ministerio de Salud. Santiago: Ministerio de Salud de Chile.

Larraín, S., Vega, J. \& Delgado, I. (1997). Relaciones familiares y maltrato infantil. Santiago: UNICEF \& Universidad de Chile.

Maslach, C. (1982). Understanding burnout: Definitional issues in analyzing a complex phenomenon. Job stress and burnout. Sage: Beverly Hills.

Morales, G. (1996). El equipo de intervención psicosocial como un grupo de alto riesgo. Salud y cambio. Revista Chilena de Medicina Social, 22, 38-45.

Morales, G. \& Lira, E. (2000). La "receta" del autocuidado: Los riesgos de equipos en programas de trabajo con violencia. En O. Vilchez (Ed.), Violencia en la cultura: Riesgos y estrategias de intervención. Santiago: Ediciones Sociedad Chilena de Psicología Clínica.

Murillo, P. (2001). El autocuidado en los psicólogos clínicos de Costa Rica: Cognitivas, conductuales y emocionales. Costa Rica: Facultad de Ciencias Sociales, Universidad de Costa Rica.

OPCIÓN. Corporación Opción (2002). Recuperado el 20 de Mayo de 2002 desde http://www.opcion.cl/presentacion1.html

Paggi, P. \& Gens, I. (2003). Síndromes traumáticos secundarios. El daño del operador en el trabajo con personas víctimas. En S. Lamberti (Ed.), Riesgos del compromiso profesional. Buenos Aires: Universidad de Buenos Aires.

Pearlman, L. A. (1999). Self-care for trauma therapists: Ameliorating vicarious traumatization. En H. Stamm (Ed.), Secondary traumatic stress. Lutherville, Maryland: Editor.

SENAME. Servicio Nacional de Menores (2002). Recuperado el 3 Marzo 2002 desde http://www.sename.cl/ maltratoinfantil/

Tapia, C. \& Iturra, V. (1996). Atención en salud. Práctica de autocuidado en profesionales de Antofagasta. Revista Horizonte de Enfermería, 7(1), 5-12.

Taylor, S. \& Bogdan, R. (1998). Introducción a los métodos cualitativos de investigación. Barcelona: Paidós.

Tonon, G. (2003). Calidad de vida y desgaste profesional. Una mirada del Sindrome del Burnout. Buenos Aires: Espacio Editorial.

Valles, M. (1999). Técnicas cualitativas de investigación social. Reflexión metodológica y práctica profesional. Madrid: Ediciones Síntesis Sociológica. 